Original Research Article

\title{
Comparison of Universal Plan Indices for Intensity Modulated Radiotherapy of Head and Neck Cancer treatment
}

\author{
B. Rekha Reddy ${ }^{1}$, M. Ravikumar ${ }^{2}$, Tanvir Pasha C.R ${ }^{3}$, Varatharaj C. ${ }^{4}$ \\ ${ }^{1}$ Smt. B. Rekha Reddy, Research Scholar, ${ }^{2}$ Dr. M. Ravikumar, Professor of Radiation Physics, ${ }^{3}$ Dr. C.R. Tanvir Pasha, \\ Associate Professor of Radiation Oncology, ${ }^{4}$ Dr. C. Varatharaj, Associate Professor of Radiation Physics, Department of \\ Radiation Physics, all authors are affiliated with Kidwai Memorial Institute of Oncology, Hosur Road, Bangalore, \\ Karnataka, India
}

Corresponding Author: Smt. B. Rekha Reddy, Research Scholar Email: rekha_289@yahoo.co.in

\begin{abstract}
Objective: Manual evaluation of IMRT plans for head-and-neck cancers has been especially challenging necessitating efficient and objective assessment tools. Based on previous clinical experience, the radiation oncologists prescribe the dose to the tumour after critical evaluation of the dose to critical structures. We have evaluated the IMRT plan using Universal Plan Indices and Quality factor. Methods and Materials: In the Eclipse Treatment Planning Systems, we have selected 15 head and neck cancer patients who underwent IMRT delivery in sliding window mode at Clinac DHX linear accelerator equipped with a 120 leaves MLC. All patients were treated using 6 MV photon beams. The UPI indices and Quality factor were calculated using HART software based on MATLAB background. Results: The mean conformity numbers for all fifteen patients were found as $0.92 \pm 0.05$ and the mean target volume ratio was $1.02 \pm 0.04$. Similarly the other indices like DG, NCI and modified HI index were $0.95 \pm 0.03,1.08 \pm 0.06$ and $0.94 \pm 0.02$ respectively. The mean overall quality factor was found to be $1.01 \pm 0.02$. The typical value of this factor also to be unity and above unity referred to be overdosed and below the unity referred as under dosage of the structures contoured volume in the corresponding plan. Conclusion: Hence, we have concluded that, evaluation of the IMRT plan of head and neck cancer patients using Universal plan Indices and Quality factor have been done successfully using HART software.
\end{abstract}

Keywords: Head and Neck Cancer, IMRT, Homogeneity Index, Conformity Number, Quality Factor

\section{Introduction}

Worldwide, head-and-neck cancer (HNC) accounts for more than 550,000 cases and 380,000 deaths annually. It is thereby the seventh most common cancer worldwide and also the seventh most common cause of death from cancer [1]. Radiotherapy plays an important role in its treatment modalities. It can be recommended as definitive treatment with or without chemotherapy, adjuvant after surgery or in the treatment of local failure after surgery. Radiotherapy allows organ preservation and improved function preservation compared to surgery and can therefore be an elegant solution [2].

The goal of radiation therapy is to deliver a lethal amount of dose to target volumes while sparing the surrounding tissues. Intensity-modulated radiation therapy (IMRT) can deliver the conformal dose distributions by varying radiation intensities within each

Manuscript received: $30^{\text {th }}$ July 2018

Reviewed: $7^{\text {th }}$ August 2018

Author Corrected: $14^{\text {th }}$ August 2018

Accepted for Publication: $18^{\text {th }}$ August 2018 field according to the fluence maps optimized by a treatment planning system (TPS). IMRT is known to improve target coverage and provide better organ-atrisk (OAR) sparing in comparison with 3D-conformal radiotherapy [3].

Manual evaluation of IMRT plans for head-and-neck cancers has been especially challenging necessitating efficient and objective assessment tools. Based on previous clinical experience, the radiation oncologists prescribe the dose to the tumour after critical evaluation of the dose to critical structures. In external beam radiotherapy, dose-volume histograms (DVH) play a key role in selecting the optimal plan for treatment delivery and it is presented in the form of cumulative DVH [4-5] and differential DVH. Homogeneity index (HI) (or uniformity index) is a tool to assess the planned dose distribution in a target volume. Despite its lack of detailed information compared to dose-volume histogram (DVH), its simplicity has made it an 


\section{Original Research Article}

attractive measure for quantifying the level of dose uniformity in a tumor volume. Several indexing formulas have been introduced in literatures [6-8]. The basic concept of these methods is to calculate the ratio of the dose value of high-dose region to the reference dose value (such as prescription dose or dose value of normal-dose region) within a target.

However, still the search is ongoing to find a single parameter to quantify the quality of a IMRT plan evaluation. Hence, we have a tried a simple method for evaluation of IMRT plan using Universal Plan Indices set and Quality Factor using Histogram Analysis in Radiation Therapy (HART), which is a MATLAB based program is an automated computational environment that was developed for the precise computation of dose-volume statistics for a large quantity of patient data used for radiation therapy research [9].

\section{Materials and Methods}

To demonstrate the IMRT plan evaluation using UPI and QF, we have selected 15 Head and Neck Cancer patients plan previously treated in DHX-Clinac Linear Accelerator (Varian Medical Systems, Palo Alto, USA) using $6 \mathrm{MV}$ photon beams. All the patients were planned on Eclipse ${ }^{\mathrm{TM}}$ treatment planning system using nine fields of equally divided gantry angles of 40 degrees. HART computation was performed on a Laptop PC with a processor speed of $2.10 \mathrm{GHz}, 4.00$ GB of RAM, and Intel Core (TM)2 Duo CPU using MATLAB (version 7.8). It uses the advanced graphical features and simulation systems available in MATLAB (Math works Inc., Natick, MA). MATLAB provides a flexible platform to set up a computational and graphical environment for other secondary software like HART. The overall quality factor $(\mathrm{QF})$ of a plan can also be determined by a linear combination of all plan indices in the UPI set.

These indices can be assessed by utilizing the DVH statistics extracted in the HART. QF can be efficiently computed for a plan by assigning the relative weights to all UPI plan indices as a complete plan evaluation strategy. Plan indices in UPI set can be systematically described as in literature [9], which includes :

a) Target coverage index (TCI).

TCI accounts for the exact coverage of PTV in a treatment plan at a given prescription dose as expressed below:

$$
\mathrm{TCI}=\frac{\mathrm{PTV}_{\mathrm{PD}}}{\mathrm{PTV}}
$$

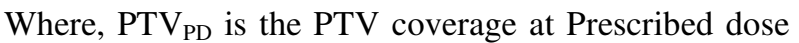
(PD), and PTV has usual meaning.

b) Critical organ scoring index (COSI).

COSI is a measure of both target coverage and critical organ overdose [10]. It can be expressed as given below:

$$
\operatorname{COSI}=\left[1-\frac{\left.\sum_{i=1}^{N} \mathrm{w}_{\mathrm{i}} \mathrm{v}(\mathrm{OAR})_{\mathrm{a}}\right)_{\mathrm{TOL}}}{\mathrm{TCI}}\right],
$$

Where, ${ }^{\mathrm{V}}(\mathrm{OAR})_{\text {Tо }}$ is the fractional volume of $\mathrm{i}^{\text {th }}$ organ at risk (OAR) receiving more than tolerance dose (TOL), and the relative weight (wi) of fractional volume of each organ is $1 / \mathrm{N}$. Where, $\mathrm{N}$ is the no. of organs.

c) Radiation conformity index (RCI).

RCI gives a consistent method for quantifying the degree of conformity based on isodose surfaces and volumes (11). It can be expressed as:

$$
\mathrm{RCI}=\frac{\mathrm{PTV}_{\mathrm{PD}}}{\mathrm{PTV}_{0.95 \mathrm{PD}}},
$$

Where, PTV $\mathrm{PV}_{0.95 \mathrm{PD}}$ is the PTV coverage at $95 \%$ of PD.

d) Prescription isodose target volume conformal index (PITV).

PITV assesses the conformity of a treatment plan [12]. However it may not be an exact parameter to identify the beam isocenter that causes a plan not to conform to the shape of the target volume in a radio-surgery treatment. PITV can be expressed as:

$$
\text { PITV }=\frac{\text { PIV }}{\text { PTV }}
$$

Where, PIV is the prescription isodose volume coverage for the target and normal tissues. PITV $>1$ and PITV $<$ 1 refers to the over treatment and under treatment regions, respectively.

e) Dose homogeneity index (HI). HI scales the "hot" spots in and around the planning target volumes $[13,14]$. It can also be expressed as:

$$
\mathrm{HI}=\frac{\mathrm{D}_{\mathrm{Max}}}{\mathrm{PD}}
$$

and modified dose homogeneity index (MHI) is defined as:

$$
\mathrm{MHI}=\frac{\mathrm{D}_{95}}{\mathrm{D}_{5}}
$$

where, DMax is the maximum dose point in PTV. Similarly D95 and D5 are the dose coverage at 95\% and $5 \%$ volume of the PTV, respectively. 


\section{Original Research Article}

f) Conformality index (CI) and conformation number $(\mathrm{CN})$. CI measures the conformity of a treatment plan. $\mathrm{CN}$ accounts for the relative measurement of dosimetric target coverage and sparing of normal tissues in a treatment plan $[15,16]$.

$$
\mathrm{CN}=\frac{\mathrm{TCI}}{\mathrm{TR}} \text {, and } \mathrm{CI}=\frac{1}{\mathrm{TR}}
$$

Where, treatment volume ratio (TR) is defined as

$$
\mathrm{TR}=\frac{\mathrm{PIV}}{\mathrm{PTV}_{\mathrm{PD}}}
$$

g) Target volume ratio (TVR).

TVR is an objective measure of how well the prescription isodose line conforms to the size and shape of the planning target volume.[16] It is simply the inverse of ratio for PITV.

$$
\text { TVR }=\frac{\text { PTV }}{\text { PIV }}
$$

h)Dose gradient index (DGI).

It examines the steepness or shallowness of dose fall off in target volume. It can be expressed as:

$$
\text { DGI }=\frac{\text { PIV }_{\mathrm{PD}}}{\mathrm{PTV}_{050 \mathrm{ID}}}
$$

Where, $\mathrm{PTV}_{0.50 \mathrm{PD}}$ is the planning target volume coverage at $50 \%$ of PD.

i) New conformity index (NCI).

NCI and HI allows for the quick and simple comparison of different radio-surgical treatment plans designed

\section{Results}

We have analyzed the Universal Plan Indices and Quality factor for IMRT plans which needs to be optimized to achieve the aim of the radiotherapy for fifteen Head and Neck cancer patients. This could help to take the final decision to go ahead with the best possible IMRT plans for the patient. The input for HART software has been acquired from Cumulative DVH data from Eclipse TPS. The final results can be visualised as a image in the graphical user interface and also in numerical values.

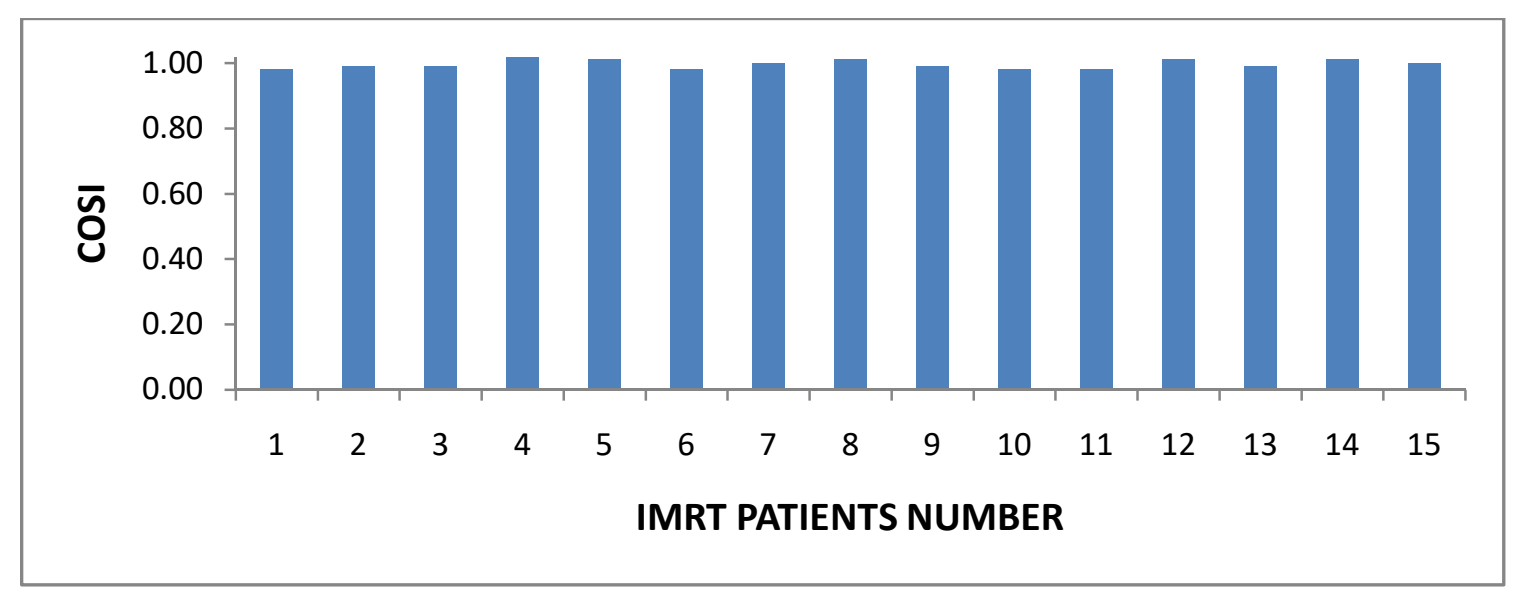

Figure-1: Comparison of COSI Index with the different IMRT patients. The mean COSI is $1.00 \pm 0.01$. 
Original Research Article

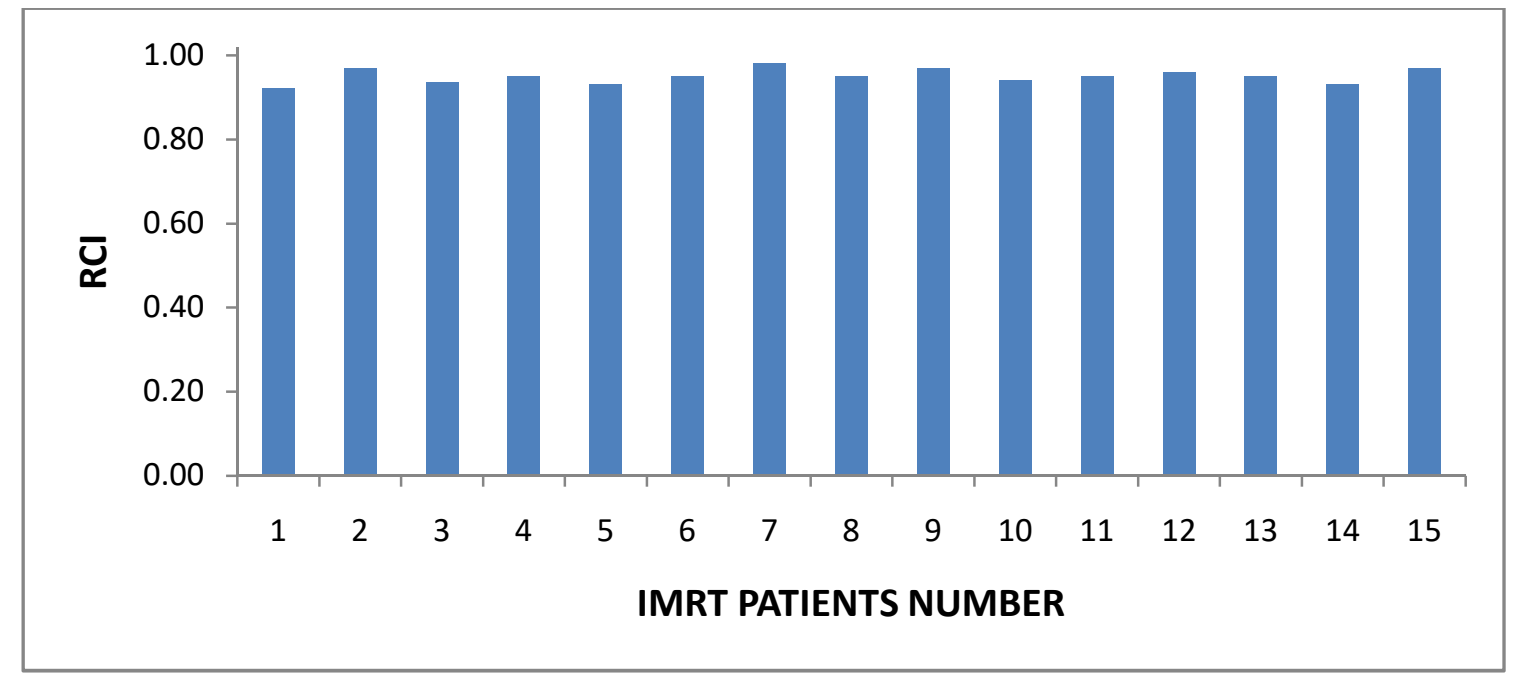

Figure-2: Comparison of RCI Index with the different IMRT patients and the mean RCI Index is $0.95 \pm 0.02$.

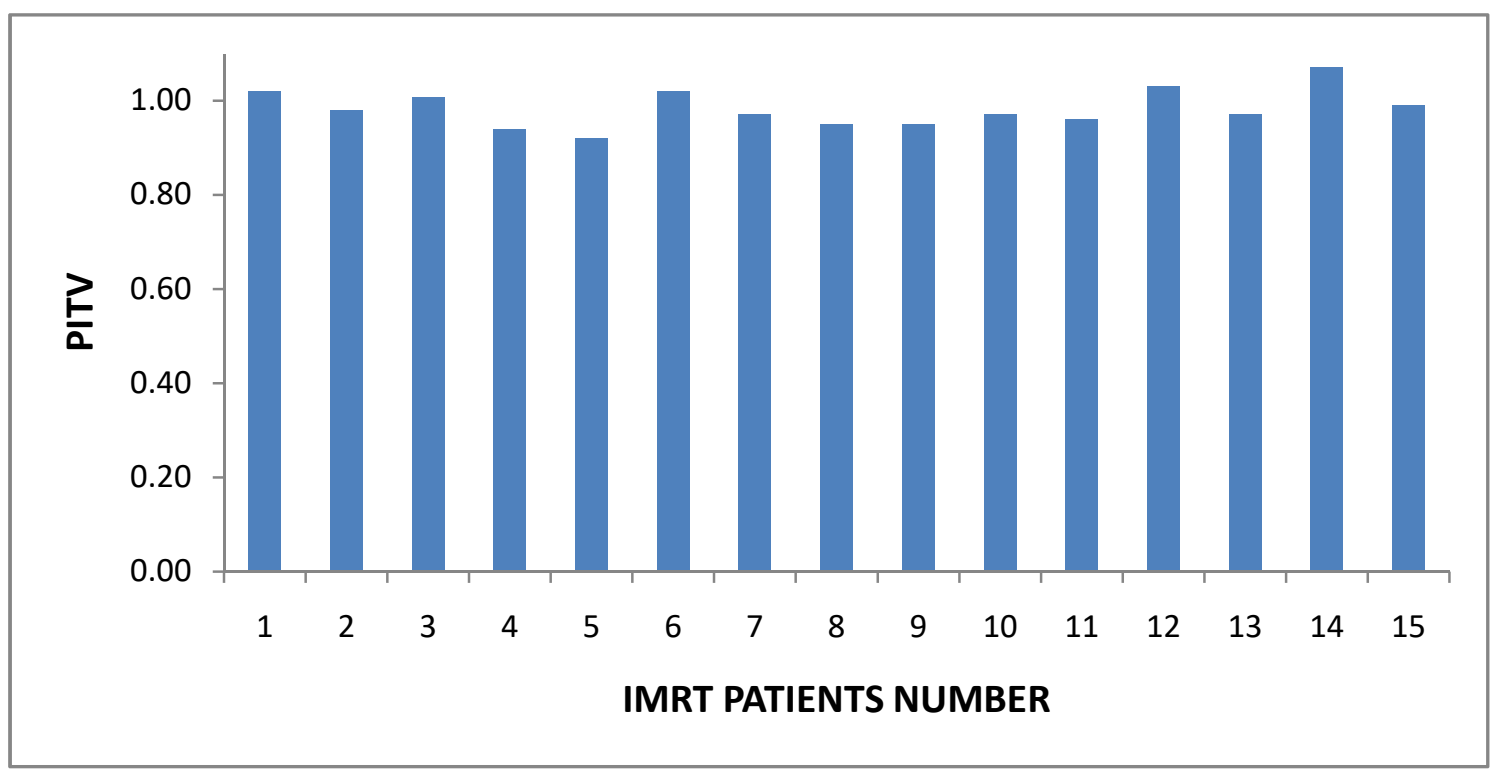

Figure-3: Comparison of PITV Index with the different IMRT patients and the mean is $0.98 \pm 0.04$.

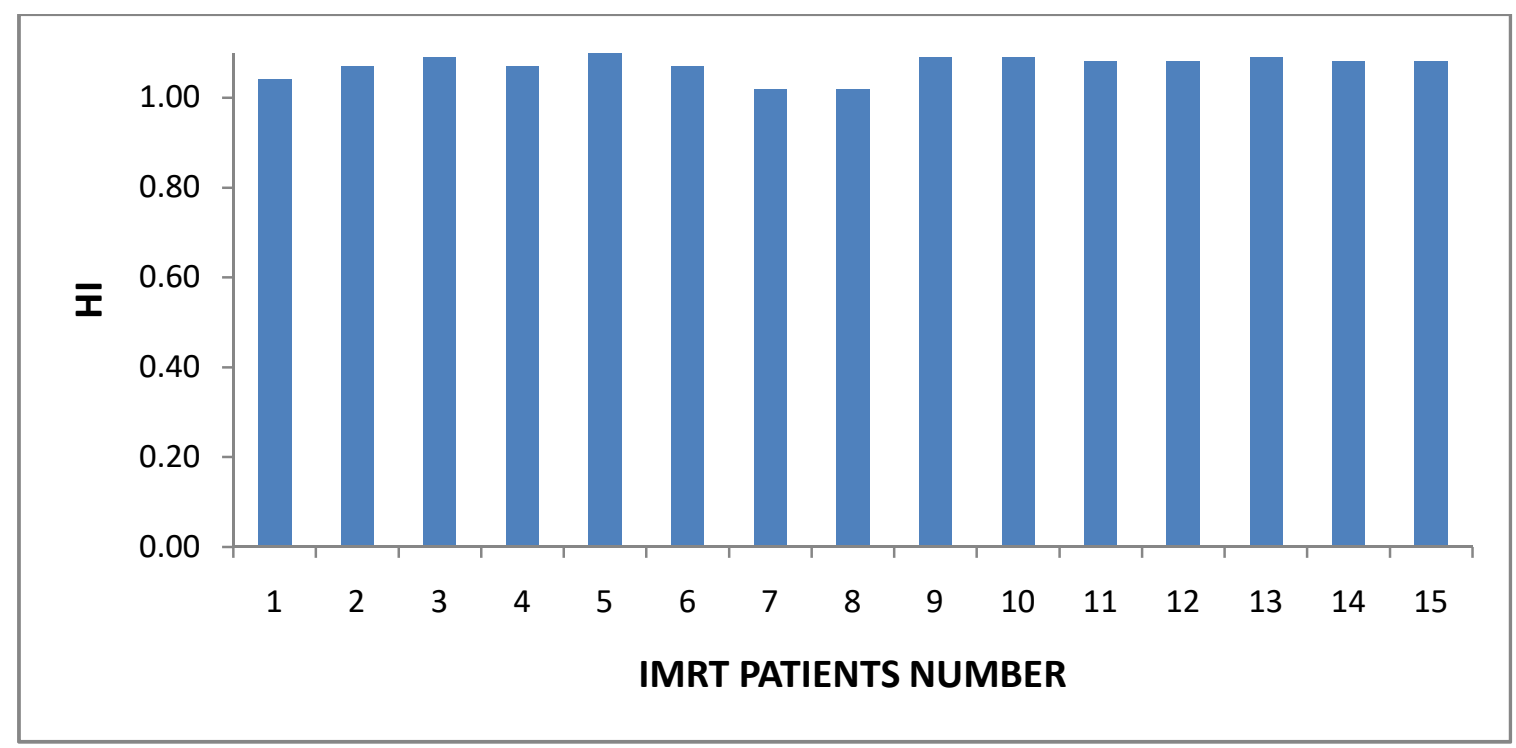

Figure-4: Comparison of Homogeneity Index with the different IMRT patients. The mean of HI is $1.07 \pm 0.03$. 


\section{Original Research Article}

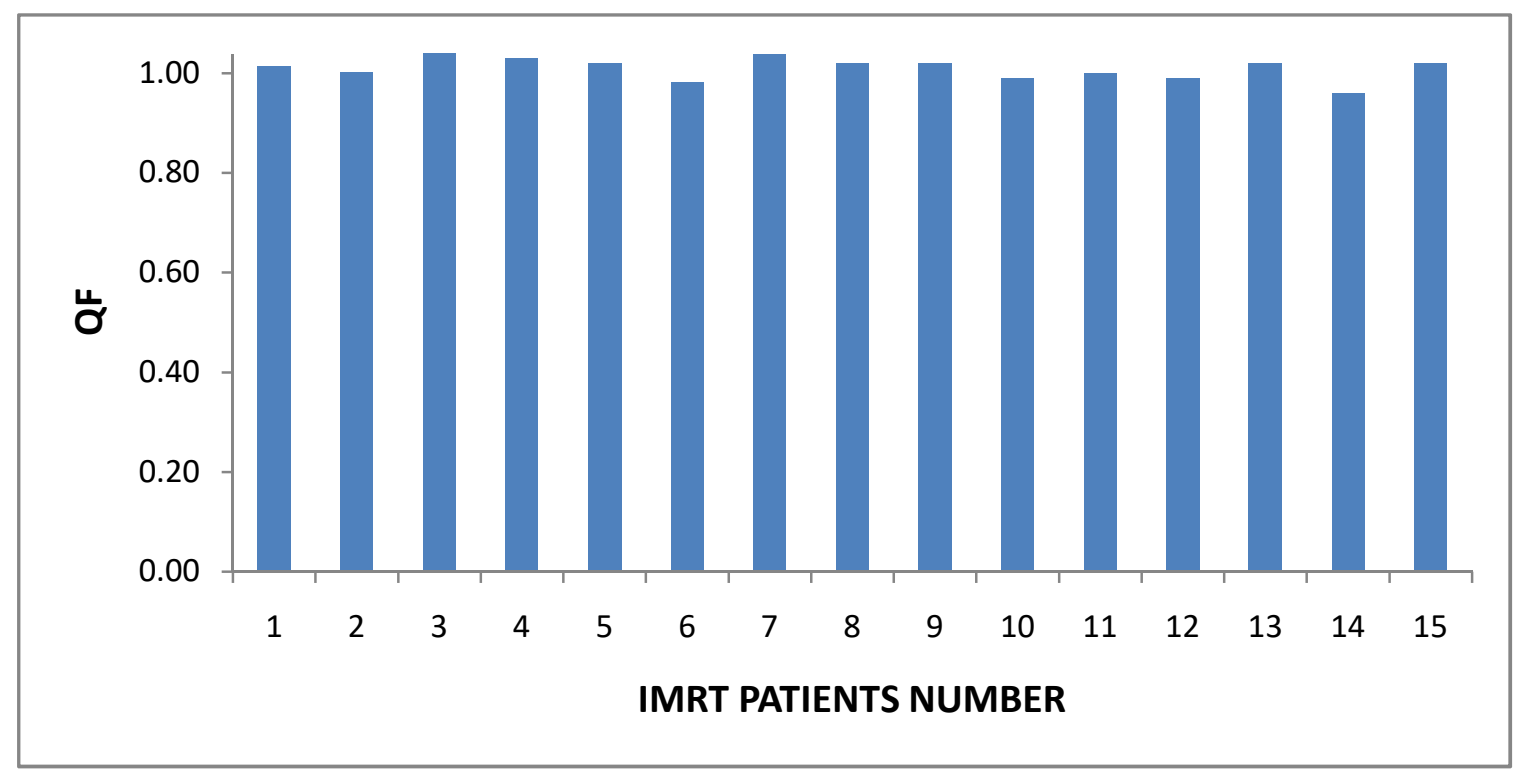

Figure-5: Comparison of overall Quality Factor with the different IMRT patients. The mean QF is $1.01 \pm 0.02$.

Table-1: Comparison of different Universal Plan Indices for fifteen Head and Neck IMRT plans

\begin{tabular}{|c|c|c|c|c|c|c|c|c|c|c|c|c|c|c|c|c|c|}
\hline \multirow[t]{2}{*}{ UPI } & \multicolumn{15}{|c|}{ Patient number } & \multirow[b]{2}{*}{ Mean } & \multirow[b]{2}{*}{ SD } \\
\hline & 1 & 2 & 3 & 4 & 5 & 6 & 7 & 8 & 9 & 10 & 11 & 12 & 13 & 14 & 15 & & \\
\hline $\mathbf{C N}$ & 0.94 & 0.97 & 0.86 & 0.93 & 0.90 & 0.86 & 0.91 & 0.86 & 0.92 & 0.78 & 0.95 & 0.92 & 0.93 & 0.78 & 0.93 & 0.92 & 0.05 \\
\hline TVR & 1.07 & 1.03 & 0.99 & 1.07 & 1.08 & 0.99 & 1.09 & 1.06 & 1.01 & 1.01 & 1.05 & 0.99 & 1.02 & 0.92 & 1.02 & 1.02 & 0.04 \\
\hline DG & 0.94 & 0.97 & 0.93 & 0.93 & 0.92 & 0.93 & 0.91 & 0.90 & 0.96 & 0.88 & 0.95 & 0.96 & 0.96 & 0.92 & 0.96 & 0.95 & 0.03 \\
\hline NCI & 1.07 & 1.03 & 1.17 & 1.07 & 1.11 & 1.17 & 1.10 & 1.16 & 1.09 & 1.27 & 1.05 & 1.09 & 1.07 & 1.29 & 1.07 & 1.08 & 0.06 \\
\hline TCI & 0.94 & 0.97 & 0.93 & 0.93 & 0.92 & 0.93 & 0.91 & 0.90 & 0.96 & 0.88 & 0.95 & 0.96 & 0.96 & 0.92 & 0.96 & 0.95 & 0.03 \\
\hline MHI & 0.95 & 0.95 & 0.94 & 0.95 & 0.92 & 0.95 & 0.93 & 0.94 & 0.94 & 0.91 & 0.93 & 0.94 & 0.94 & 0.94 & 0.95 & 0.94 & 0.02 \\
\hline
\end{tabular}

Figure 1 shows the comparison of COSI Index value for the fifteen patients and the Figure 2 compare the RC Index. The similar comparison of PITV and Homogeneity Index were shown in Figure 3 and 4. The other indices and their mean values with standard error were shown in the Table.1. The mean conformity numbers for all fifteen patients were found as $0.92 \pm 0.05$ and the mean target volume ratio was $1.02 \pm 0.04$. Similarly the other indices like DG, NCI and modified HI index were $0.95 \pm 0.03,1.08 \pm 0.06$ and $0.94 \pm 0.02$ respectively. The overall quality factor for all the cases were shown in figure 5. The minimum, maximum QF values were found to be 0.96 and the 1.06 , where as the mean QF was found to be $1.01 \pm 0.02$.

\section{Discussion}

Prabhkar et al. [18] proposed a index, PNI, which gives a quick comparison of the plan that results in reduced dose to the critical structures and totally relies on the tolerance doses to the critical structures and can be used as an additional tool for routine treatment plan evaluation. In other study by Jayapalan et al [19] have evaluated the impact of conformity index in the unified dosimetry index (UDI) score for two different planning techniques namely intensity-modulated radiotherapy (IMRT) and Rapid Arc and concluded that Rapid Arc plans scored better UDI value as well as better OARs sparing. In the comparison of IMRT and Rapid Arc plans using the UDI score, the impact of conformity index was significant.

Wu et al. [20] evaluated the association of Conformity Index with the size and shape of the target volume and they found that, CI has tend to have inferior values for smaller or more complex targets as compared to those with larger volumes or simpler shapes. In other study by Collins et al [21] have evaluated the relation between shape, size and complexity of skull base tumours with 


\section{Original Research Article}

parameters like new $\mathrm{CI}, \mathrm{HI}$ and found that these parameters were independent of each other in particular to cyberknife treatment.

All these studies were more focused on analyzing the conformity and homogeneity indices. In our present analysis, as there were no single tool or parameter found yet to analyse the pal, we have focused to find Universal plan indices and Quality Factor based evaluation. As mentioned by the Pyakuryal et al., [9], the ideal value of the all the plan indices calculated above should be unity. But these indices alone cannot act as reference; hence we have used all these indices together and found the Quality Factor for each delivered plan. The typical value of this factor also to be unity and above unity referred to be overdosed and below the unity referred as under dosage of the structures contoured volume in the corresponding plan.

\section{Conclusion}

We have successfully evaluated the IMRT plan of head and neck cancer patients using Universal plan Indices and Quality factor. These factors will be very much useful for all types of radiotherapy plan evaluation method to identify the better possible plan to deliver maximum dose to the tumour cells and the same time achieving less dose the normal cells. These Indices also can be used to compare between the different plans of the same patient in the treatment planning systems so that, the best plan can be delivered.

\section{Funding: Nil, Conflict of interest: None Permission of IRB: Yes}

\section{References}

1. Global Burden of Disease Cancer Collaboration, Fitzmaurice $\mathrm{C}^{1}$, Allen $\mathrm{C}^{2}$, Barber $\mathrm{RM}^{2}$, Barregard L, et al. Global, Regional, and National Cancer Incidence, Mortality, Years of Life Lost, Years Lived With Disability, and Disability-Adjusted Life-years for 32 Cancer Groups, 1990 to 2015: A Systematic Analysis for the Global Burden of Disease Study. DOI:10.1001/ jamaoncol.2016.5688

2. O'Sullivan B , Rumble RB, Warde P; et al. Intensitymodulated radiotherapy in the treatment of head and neck cancer. DOI:10.1016/j.clon.2012.05.006

3. Zelefsky MJ, Yamada Y, Fuks Z, et al. Long-term results of conformal radiotherapy for prostate cancer: impact of dose escalation on biochemical tumor control and distant metastases-free survival outcomes. DOI:10. 1016/j.ijrobp.2007.11.066
4. Austin-Seymour MM, Chen GT, Castro JR, et al. Dose volume histogram analysis of liver radiation tolerance. Int J Radiat Oncol Biol Phys. 1986 Jan;12 (1): $31-5$.

5. Lawrence TS, Tesser RJ, ten Haken RK. An application of dose volume histograms to the treatment of intrahepatic malignancies with radiation therapy. Int J Radiat Oncol Biol Phys. 1990 Oct; 19(4): 1041-7.

6. Shaw E, Kline R, Gillin M, et al. Radiation Therapy Oncology Group: radiosurgery quality assurance guidelines. Int J Radiat Oncol Biol Phys. 1993 Dec 1;27 (5): 1231-9.

7. Wu Q, Mohan R, Morris M, et al. Simultaneous integrated boost intensity-modulated radiotherapy for locally advanced head-and-neck squamous cell carcinomas. I: dosimetric results. Int J Radiat Oncol Biol Phys. 2003 Jun 1;56 (2):573-85.

8. Wang X, Zhang X, Dong L, et al. Effectiveness of noncoplanar IMRT planning using a parallelized multiresolution beam angle optimization method for paranasal sinus carcinoma. DOI:10.1016/j. ijrobp. 2005. 06.006

9. Pyakuryal A, Myint WK, Gopalakrishnan M, et al. A computational tool for the efficient analysis of dosevolume histograms from radiation therapy treatment plans. J Appl Clin Med Phys. 2010 Jan 28;11(1):3013.

10. Menhel J, Levin D, Alezra D, et al. Assessing the quality of conformal treatment planning: a new tool for quantitative comparison. DOI:10.1088/ 0031-9155/51/ 20/019

11. Knöös T, Kristensen I, Nilsson P. Volumetric and dosimetric evaluation of radiation treatment plans: radiation conformity index. Int J Radiat Oncol Biol Phys. 1998 Dec 1;42(5):1169-76.

12. Leung LH, Chua DT, Wu PM. A new tool for dose conformity evaluation of radiosurgery treatment plans. Int J Radiat Oncol Biol Phys. 1999 Aug 1;45(1):233-41.

13. Akpati H, Kim C, Kim B, et al. Unified dosimetry index (UDI): a figure of merit for ranking treatment plans. J Appl Clin Med Phys. 2008 Jun 23;9(3):2803.

14. Yoon M, Park SY, Shin D, et al.Anew homo-geneity index based on statistical analysis of the dose-volume histogram. J Appl Clin Med Phys. 2007 Mar 20; 8 (2):9-17. 
15. Leung LH, Kan MW, Cheng AC, et al. A new dosevolume-based Plan Quality Index for IMRT plan comparison. DOI:10.1016/j.radonc.2007.10.018

16. Paddick I. A simple scoring ratio to index the conformity of radiosurgical treatment plans. Technical note. DOI:10.3171/jns.2000.93.supplement

17. Collins SP, Coppa ND, Zhang Y, et al. Cyber Knife radiosurgery in the treatment of complex skull base tumors: analysis of treatment planning parameters. DOI: 10.1186/1748-717X-1-46

18. Prabhakar R, Rath GK. A simple plan evaluation index based on the dose to critical structures in radiotherapy. DOI:10.4103/0971-6203.89965
Original Research Article

19. Krishnan J, Shetty J, Rao S, et al. Comparison of Rapid Arc and Intensity-modulated Radiotherapy Plans Using Unified Dosimetry Index and the Impact of Conformity Index on Unified Dosimetry Index Evaluation. DOI:10.4103/jmp.JMP_112_16

20. Semenenko VA, Reitz B, Day E, et al. Evaluation of a commercial biologically based IMRT treatment planning system. DOI:10.1118/1.3013556

21. Wu QR, Wessels BW, Einstein DB, et al. Quality of coverage: conformity measures for stereotactic radiosurgery. DOI:10.1120/1.1621372

\section{How to cite this article?}

B. Rekha Reddy, M. Ravikumar, Tanvir Pasha C.R, Varatharaj C. Comparison of Universal Plan Indices for Intensity Modulated Radiotherapy of Head and Neck Cancer treatment. Int J Med Res Rev 2018; 6(06): 321-327.doi: 10.17511/ ijmrr. 2018. i06.05. 